\title{
A newly identified mutation in prion protein is associated with diarrhoea and autonomic neuropathy
}

Human prion diseases are caused by propagation of abnormal prion protein, and are usually characterized by rapid neurodegeneration, resulting in neurological and neuropsychiatric symptoms. A team led by John Collinge at the MRC Prion Unit, Institute of Neurology, London has identified a novel prion protein $(P R N P)$ gene mutation that causes prion protein accumulation in multiple peripheral tissues, and initially manifests as diarrhoea, autonomic failure and peripheral neuropathy, with CNS symptoms emerging later in life.

\section{1 ...this study describes a} distinct ... phenotype with a nonCNS presentation... 77

Approximately $15 \%$ of all instances of prion disease are inherited, and identification of the underlying mutations could help researchers to understand the pathophysiology of these disorders.
Collinge and his team studied a large kindred in the UK who had unique clinical symptoms. While in their $30 \mathrm{~s}$, the patients developed chronic diarrhoea, which was closely followed by symptoms of a mixed sensory and autonomic neuropathy. Cognitive problems and seizures developed when the patients were in their 40 s or 50 s, and average age at death was 57 years.

By sequencing genomic DNA from the affected patients, the researchers identified an autosomal dominant genetic mutation in PRNP that results in Tyr163X, which in turn causes a truncated protein.

Neuropathological analysis of autopsy and biopsy samples from these individuals revealed accumulation of abnormal prion protein in peripheral organs such as the liver and duodenum. Brain tissue showed mild spongiosis with amyloid plaques and neurofibrillary tangles. In the spinal cord, nerve fibres and blood vessels in the dorsal root ganglia had abundant prion protein deposits.

Immunoblots of brain homogenates confirmed that these atypical features were the result of the mutated protein, which has an apparent molecular mass greater than the normal full-length protein. The disease was not transmissible to mice via inoculation with brain tissue.

This study describes a distinct prion disease phenotype with a non-CNS presentation and widespread deposition of prion protein in systemic organs. The slow disease progression and predominantly peripheral symptoms suggests that this prion disease may be difficult to diagnose and should be consisered in patients with unexplained chronic diarrhoea and neuropathy.

\section{Ellen Bible}

Original article Mead, S. et al. A novel prion disease associated with diarrhea and autonomic neuropathy. N. Engl. J. Med. doi: 10.1056/NEJMoa1214747 